\title{
Changes in Concentration of Hypothalamic Cytosolic Progestin Receptors at the Initiation of Pseudopregnancy in Rats
}

\author{
Michio TAKAHASHI AND Atsuko SAITO \\ Laboratory of Veterinary Physiology, Faculty of Agriculture, \\ University of Tokyo, 1-1-1 Yayoi Bunkyo-ku, Tokyo 113
}

\begin{abstract}
Pseudopregnancy (psp) can be induced by a single injection of progesterone in cyclic rats and the administration of anti-progesterone serum can block the establishment of $\mathrm{psp}$ in cervically stimulated rats. To further investigate neuroendocrine mechanisms for the initiation of either psp or prolactin (PRL) surges in connection with the neurotropic action of progesterone, cytosolic progestin receptors (PRc) were identified and measured in preoptic (POA) and basal hypothalmic $(\mathrm{BH})$ areas.

Chronological determinations of PRc concentrations in both areas revealed large fluctuations in the morning of the estrous day but not in the morning of the diestrous day. The differences between the maximal and the minimal PRc concentrations observed were more than 100 -fold in POA and 3 -fold in BH. Cervical stimulation applied in the afternoon of the proestrous day significantly altered the changing pattern of PRc concentrations in POA but not in BH. One of the two peaks of PRc concentrations in POA was magnified and advanced earlier to $0300 \mathrm{~h}$, and then the 1st PRL surge peaking at $0700 \mathrm{~h}$ occurred. This PRL release seemed to stimulate progesterone secretion and an elevation of peripheral progesterone levels coincided with the 2nd peak of PRc concentrations in POA at $0900 \mathrm{~h}$. This coincidence may be a prerequisite for the further continuation of PRL surges.

These results strongly suggest that the spontaneous oscillation of the PRc concentration in hypothalamic neurons is involved in the regulation of PRL secretion and that cervical stimulation shifts the phase and changes the amplitude.
\end{abstract}

Pseudopregnancy (psp) in the rat under a daily light-dark photo cycle is a consequence of two daily prolactin (PRL) surges (Smith and Neill, 1976). PRL surges can be induced by either uterine cervical stimulation or administration of progesterone. Progesterone is to be admistered once in a large amount (e.g. $10 \mathrm{mg}$, Murakami et al., 1980 ) or for a few days at a dose sufficient to maintain peripheral progesterone at luteal phase levels (Everett, 1963).

\section{Received April 25, 1983.}

This article is dedicated to the memory of Professor M. Kawakami, who wholeheartedly served as the chief editor of Endocrinologia Japonica.
The first PRL surge after cervical stimulation occurs within a limited period in the early morning of the estrous day (0500-0700 h). Moreover, single administration of progesterone in a large amount is only effective in inducing psp when the injection is done at 0700 to $0800 \mathrm{~h}$ of the estrous day, and a shift of injection time more than $2 \mathrm{~h}$ nullifies the effect (Murakami et al., 1980). These time-dependent phenomena suggest the existence of a neural apparatus which perceives peripheral progesterone levels and is involved in the appearance and the maintenance of PRL surges.

The present study describes chronological 
changes in hypothalamic cytosolic progestin receptor $(P R c)$ at the initial stage of $p s p$ in relation to peripheral hormonal levels, particularly those of PRL.

\section{Materials and Methods}

\begin{abstract}
Animals
Adult Wistar-Imamichi rats kept in a light and temperature $\left(23 \pm 2^{\circ} \mathrm{C}\right)$ controlled room under a light regimen of 14L:10D (lights on $0500-1900 \mathrm{~h}$ ) were used. Vaginal smears were taken every day; only animals showing a 4 day cycle for more than 2 weeks were used. Three groups of rats, cycling, cervically stimulated, and estrogen-primed were provided. Cervical stimulation was applied mechanically with a glass rod between $1800-1900 \mathrm{~h}$ on the proestrous day. Estrogen-priming to induce PRc (Moguilesky and Raynaud, 1979) was done by sc injections of estradiol benzoate (EB; $20 \mu \mathrm{g} /$ day) starting at estrus once (a) or for 3 days (b). The Scathchard analyses of hypothalamic (a) and uterine PRc ( $a$ and $b$ ) were performed 2 days after the end of estrogen-priming.
\end{abstract}

\section{Tissue dissection}

Animals were decapitated and trunk blood was collected. The uteri were stripped of adhering fat and mesentery and minced. Uteri from 3 rats were pooled. The dorsal plane of the brain was fixed on a glass plate cooling down to $-20^{\circ} \mathrm{C}$ with a thermomodule. The medial preoptic area (POA) and the mediobasal hypothalamus (BH) were dissected out using a razor blade and fine forceps as described by Lauine et al. (1974). The weight of a block ranged 20-25 mg and 5 blocks were pooled for a sample for the PRc assay. The hypothalamic blocks were collected from either cycling or cervically stimulated rats through an estrous day and a day after cervical stimulation at 0000, 0300,0500,0700, 0900, and $1200 \mathrm{~h}$. For each point, 3-4 samples were analyzed. A part of randomly selected 5-10 blood samples collected simultaneously with the sampling of hypothalami were analysed for serum progesterone, $17 \beta$-estradiol and PRL concentrations. Other hypothalamic samples were collected from cycling rat with the same time schedule on the metestrous day.

\section{Preparation of cytosol and PRc assays}

The cyctosol fractions from the tissues were obtained according to the method described by MacLusky and McEwen (1980). Homogenization volumes were $100 \mathrm{ml} / \mathrm{g}$ for the uteri and $12 \mathrm{ml} / \mathrm{g}$ for the hypothalami. The homogenates were centrifuged in a model IEC/B-60 ultracentrifuge (Damon/IEC Division,
Needham Heights, MA) at $0^{\circ} \mathrm{C}$ for $1 \mathrm{~h}$ at $105,000 \mathrm{xg}$. The supernatant cytosol fractions were decanted and used immediately. Protein concentrations were determined by the method of Lowry et al. (1951) using bovine serum albumin (fraction $\mathrm{V}$, essentially fatty acid-free, Sigma Chemical Co., Saint Louis, MO). Protein concentrations ranged $3.5-4.5 \mathrm{mg} / \mathrm{ml}$ (uterine samples) or $0.9-1.6 \mathrm{mg} / \mathrm{ml}$ (hypothalamic samples).

The exchange assays for PRc using R5020 as a high affinity ligang (Raynaud, 1977) were performed mainly based on the method by Maclusky and McEwen (1980). Aliquots $(200 \mu 1)$ of cytosol fraction were incubated at $0^{\circ} \mathrm{C}$ for $4 \mathrm{~h}$ with $\left[{ }^{3} \mathrm{H}\right] \mathrm{R} 5020(17 \alpha$ methyl- $\left[{ }^{3} \mathrm{H}\right] \mathrm{R} 5020,87 \mathrm{Ci} / \mathrm{mmol}$, New England Nuclear, Boston MA) dissolved in $100 \mu 1$ TEGD buffer [for TEGD buffer, see MacLasky and McEwen (1980)]. Macromolecular bound and free $\left[{ }^{3} \mathrm{H}\right] \mathrm{R} 5020$ were separated by the procedure described originally by Ginsberg et al. (1974) and modified by MacLusky and McEwen (1980). Samples $(250 \mu 1)$ of each incubate were applied to columns $(0.5 \times 7 \mathrm{~cm})$ of LH20 (Pharmacia Fine Chemicals, Piscataway, NY) equilibriated with TEGD buffer at $2-4^{\circ} \mathrm{C}$. The samples were washed in with buffer $(150 \mu 1)$ and allowed to stand for $30 \mathrm{~min}$. Macromolecular bound $\left[{ }^{3} \mathrm{H}\right] \mathrm{R} 5020$ was eluted with $0.8 \mathrm{ml}$ buffer and the last $0.4 \mathrm{ml}$ was put into a scintillation vial and counted with $10 \mathrm{ml}$ toluene-Liquifluor-TritonX100 [917 $\mathrm{ml}$ toluene, $83 \mathrm{ml}$ Liquifluor (New England Nuclear), and $500 \mathrm{ml}$ TritonX100 (New England Nuclear)] in a scintillation spectrometer (Packard, Downers Grove, IL).

Scatchard analyses of uterine and hypothalamic PRc

Aliquots $(200 \mu 1)$ of cytosol were incubated with a range of concentration of $\left[{ }^{3} \mathrm{H}\right] \mathrm{R} 5020$ dissolved in TEGD buffer $\left(100 \mu 1\right.$; final $\left[{ }^{3} \mathrm{H}\right] \mathrm{R} 5020$ concentrations, $0.45-28.6 \mathrm{nM}$ ) overnight at $0^{\circ} \mathrm{C}$. One of 2 series incubations was carried out with 100 -fold molar excess of unlabeled R5020. Incubates were chromatographed on LH-20, as described above. Results were analysed by the method of Scatchard (1949).

\section{One-point assay of hypothalamic PRc}

Aliquots $(200 \mu 1)$ of POA and $\mathrm{BH}$ cytosol obtained at various times during different reproductive phases were incubated for $4-16 \mathrm{~h}$ with $\left[{ }^{3} \mathrm{H}\right] \mathrm{R} 5020$ in TEGD buffer $(100 \mu 1)$ (final $\left[{ }^{3} \mathrm{H}\right] \mathrm{R} 5020$ concentration, 14.3 $\mathrm{nM}$ ) in the presence or absence of $1.43 \mu \mathrm{M}$ unlabeled R5020. Different incubation length within the range described above was not found to influence the final results. Bound radioactivity in samples $(250 \mu 1)$ of the incubates was determined by Sephadex LH-20 gel filtration. Limited capacity of binding (specific binding), obtained as the difference in binding between incubated with and without unlabeled R5020, 
was expressed as per mg cytosol protein.

\section{Hormone assays}

Hormones were radioimmunoassayed as described elsewhere; progesterone and PRL (Murakami et al., 1979), and $17 \beta$-estradiol (Nishihara and Takahashi, 1983).

\section{Results}

Bindig affinity and capacity of PRc of uterine and hypothalamic cytosol (Fig. 1)

Scatchard presentations of isotherms constructed for $\left[{ }^{3} \mathrm{H}\right] \mathrm{R} 5020$ binding in uterine and hypothalamic (only POA samples were used) cytosols from estrogen primed rats revealed a class of binding sites with a similar

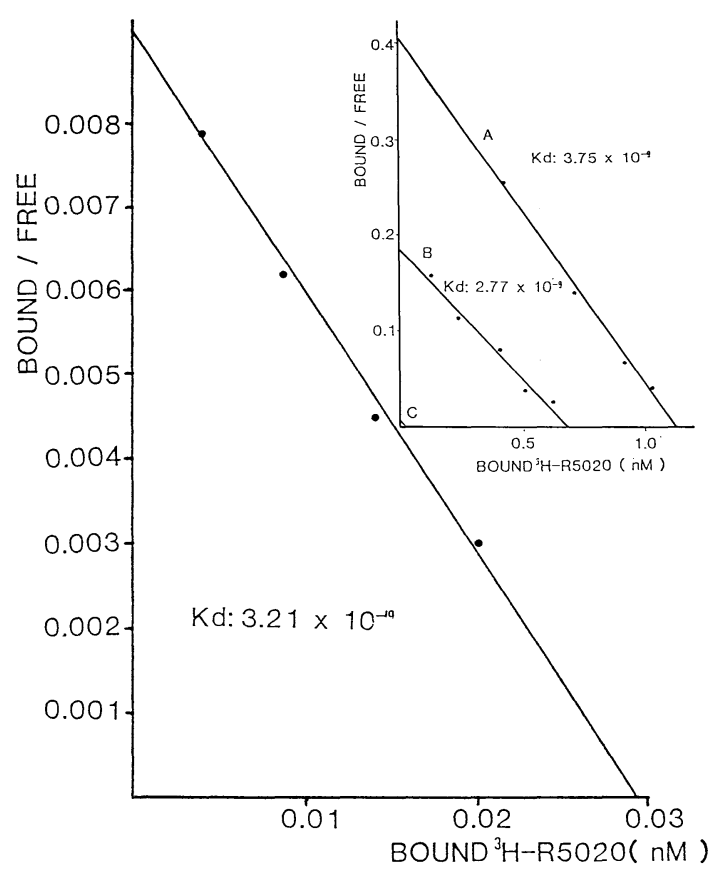

Fig. 1. Scatchard analysis of specific $\left[{ }^{3} \mathrm{H}\right] \mathrm{R} 5020$ binding to cytosol from medial preoptic area (POA). For the reference, the results of uterine cytosols $(\mathrm{A}, \mathrm{B})$ are presented in the inset together with the results of POA with the same scale (C). Animals were pretreated with estradial benzoate for 3 days $(A)$ or 1 day $(B, C)$. Note that the number of binding sites/mg protein in POA cytosols is far less than that in uterine cytosols. apparent dissociation constant $(\mathrm{Kd})$ of approximately $0.3-0.4 \mathrm{nM}$. The $\mathrm{Kd}$ values of hypothalamus cytosolic PRc were in good accordance with a previous one reported as a higher affinity component of 2 classes of PRc in hypothalamic cytosol (Maclusky and McEwen, 1980) and that of uterine cytosolic PRc was slightly lower that reported by Attardi and Palumbo (1981).

Comparisons of uterine cytosols from rats treated with $\mathrm{EB}$ for 1 and 3 days indicated a substantial increase in the number of available high affinity sites by EB priming. Concentration of high affinity sites in the hypothalamic cytosol from EB primed (1 day) rats was approximately $1 / 25$ of those in the uterine cytosol.

Hypothalamic PRc concentrations during the mornings of the estrous and diestrous day, and the effect of cervical stimulation.

Chronological changes in PRc concentrations both in POA (Fig. 2) and $\mathrm{BH}$ (Fig. 3)

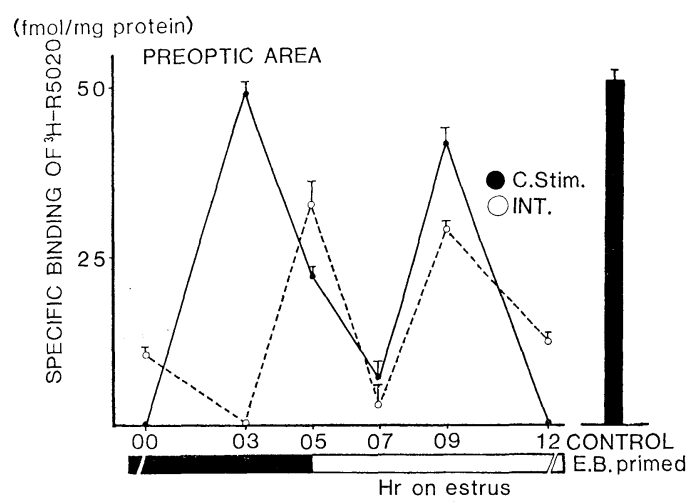

Fig. 2. Chronological changes in the specific R5020 binding to the cytosols from medial preoptic area (POA) during the morning of the estrous day. Intact (INT. : open circle) rats were cycling regularly. Cervical stimulation (C. Stim.: closed circle) was applied at $1800 \mathrm{~h}$ on the proestrous day. Vertical column represents the value obtained in rats pretreated once with estradiol benzoate. Each point and vertical bar represents the mean \pm S. E. of 3 to 4 samples. Each samples consisted of 5 hypothalami. Horizontal column at the bottom indicates a dark (closed) and a lightning (open) phase. 


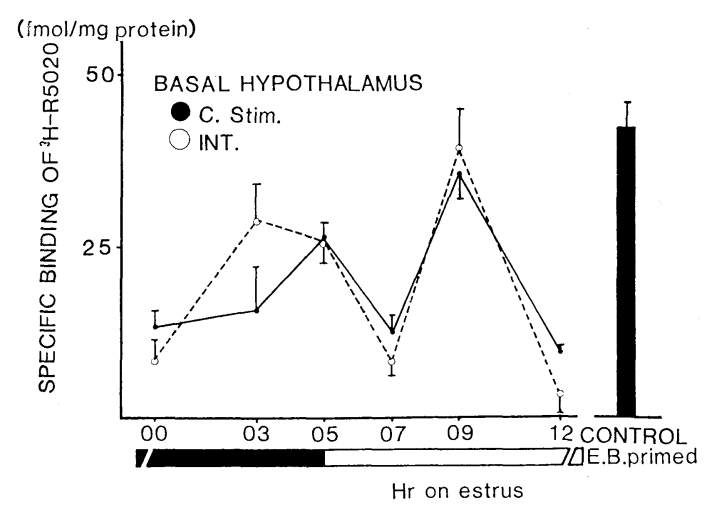

Fig. 3. Chronological changes in the specific R5020 binding to the cytosol from mediobasal hypothalamic area $(\mathrm{BH})$ during the morning of the estrous day. For further explanation, see Fig. 2.

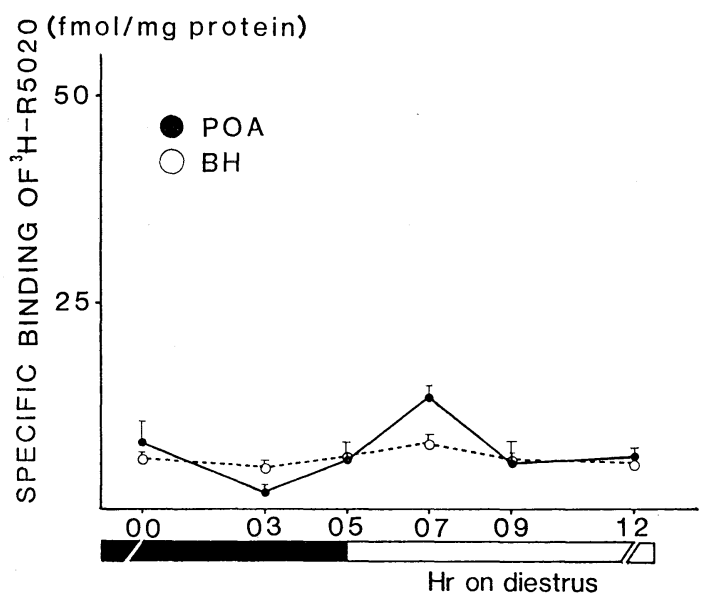

Fig. 4. Chronological changes in the specific R5020 binding to the cytosols from either mediobasal hypothalamic area (open circle: $\mathrm{BH}$ ) or medial preoptic area (closed circle: POA) during the morning of the diestrous day (a day after estrus). For further explanation, see Fig. 2.

were remarkable on the estrous day. The maximum levels in each hypothalamic area were almost equivalent to those observed in the corresponding areas of EB-primed rats. The difference between the lowest and the highest PRc concentrations was more than 100-fold in POA and approximately 3-fold in $\mathrm{BH}$. Under our sampling schedule, there were observed 2 peaks at 0500 and $0900 \mathrm{~h}$ for POA and at 0300 and $0900 \mathrm{~h}$ for $\mathrm{BH}$.
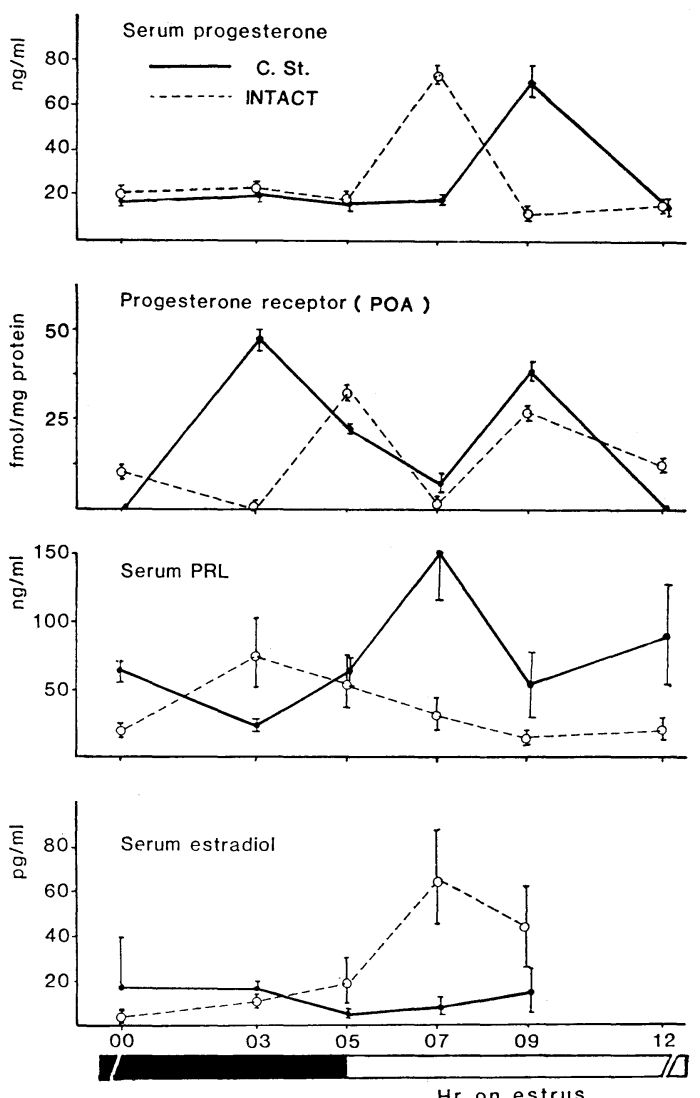

Fig. 5. Chronological changes in hormonal concentrations in peripheral sera during the morning of the estrous day. The results for progestin receptors in preoptic area (POA) are taken from Fig. 2. Each point and vertical bar in the hormonal measurements represents the mean \pm S.E. of 5 to 10 samples. No determination of estradiol was made at $1200 \mathrm{~h}$.

Fluctuation of PRc concentrations disappeared almost completely on the diestrous day (a day after estrus) (Fig. 4).

Cervical stimulation advanced and enlarged the earlier peak in POA (Fig. 2). The pattern of the changes was virtually unaltered in $\mathrm{BH}$ by cervical stimulation (Fig. $3)$.

Changes in serum hormones levels in intact and cervically stimulated rats on the estrous day (Fig. 5). 
A temporal increase in the progesterone concentration was observed at $0700 \mathrm{~h}$ in intact rats. The occurrence of this increase seemed to be delayed $2 \mathrm{~h}$ in cervically stimulated rats.

The first nocturnal PRL surge in cervically stimulated rats was detected in $0700 \mathrm{~h}$ samples. Fluctuations in PRL concentrations were not evident in intact rats.

A rise in $17 \beta$-estradiol levels later than $0700 \mathrm{~h}$ in intact rats was suppressed in cervically stimulated rats.

In intact rats, the 1 st $(0500 \mathrm{~h})$ and the 2nd $(0900 \mathrm{~h})$ increase in the number of PRc occurred when progesterone levels stayed at low levels. In cervically stimulated rats, the second rise in PRc $(0900 \mathrm{~h})$ was synchronized with the rise in progesterone levels. The PRL rise in cervically stimulated rats occurred after the 1st PRc rise which was followed by a progesterone rise.

\section{Discussion}

Electrolytic lesions of the medial preoptic area of cyclic rats induce a spontaneous consecutive psp (Clemens et al., 1976), and this area is considered to possess neurons which inhibit the expression of nocturnal PRL surges (Freeman and Banks, 1980). Various parts of the hypothalamus including the POA are reported to have specific PRc (Kato and Onouchi, 1979; Maclusky and McEwen, 1980; Attardi and Palumbo, 1981). If progesterone could bind to POA neurons and inhibit their activity, the nocturnal surges will ensue and therefore, psp will be induced.

The results of sequential determination of PRc concentrations in POA and $\mathrm{BH}$ in the morning of the estrous day revealed extraordinary large fluctuations particularly in POA. The occurrence of these fluctuations was suggested to be a specific event on the estrous day, for there were virtually no fluctuations one day later. The critical period when the progesterone treatment was effective in inducing psp in cyclic rats $(0700-$ $0800 \mathrm{~h}$ on the day of estrus; Murakami et al., 1980) corresponded to the period when PRc stayed at a very low level. This period was also characterized by the highest peripheral progesterone concentration throughout the estrous day morning. But this elevation of the endogenous progesterone level might not adequately be perceived by POA neurons, because of the low availability of PRc at this period. The neuronal inability specific to this period would prevent the release of PRL and secure the recurrence of the estrous cycle. In contrast, the administration of progesterone during the critical period with a large pharmacological dose immediately induces a maximal release of PRL lasting for at least $4 \mathrm{~h}$ (Murakami et al., 1980). If PRc of POA, though small in number, was fully saturated, progesterone could exert its effect on POA neurons and eliminate their inhibitory effect on PRL surges.

The application of cervical stimulation at $1800 \mathrm{~h}$ one day before did not greatly alter the pattern of change in PRc concentrations in $\mathrm{BH}$. In POA, however, the earlier of the two rises in the PRc concentration was advanced and magnified. The earlier rise occurred at $0300 \mathrm{~h}$ when the peripheral progesterone level was low. In view of the change in the PRc concentration, POA was considered to be most sensitive to progesterone at this period, which would enable the POA neurons to receive a low level of progesterone as a significant biological signal. It is known that cervical stimulation in ovariectomized rats also induces PRL surges (Freeman et al., 1974). Before we conclude that the mechanism mentioned above is involved in the occurrence of the 1st PRL surge, we have to do more similar experiments to this study using ovariectomized rats.

We reported previously (Murakami et al., 1982) that luteal cells harvested from cyclic 
rats between the estrous and the morning of the late diestrous day responded to the stimulation of PRL with a significant increase in progesterone production. An elevation of progesterone level at $0900 \mathrm{~h}$ in cervically stimulated rats in this study could be attributed to the antecedent occurrence of PRL surge at $0700 \mathrm{~h}$. Thus, the second rise in the PRc concentration in cervically stimulated rats coincided with the endogenous progesterone rise. Since preclusion of this progestrone rise by an injection of antiprogesterone serum at $0700-0800 \mathrm{~h}$ on the estrous day resulted in the suppression of the diurnal PRL surge expected to occur around $1800 \mathrm{~h}$ following the 1 st nocturnal surge (Takahashi et al., 1980), this coincidence of progesterone and PRc rises may be necessary to continue the recurrence of PRL surges.

As we confirmed in this study, it is well accepted that estrogen leads to the induction of PRc in the hypothalamus as well as the pituitary and in the tissues of the reproductive tract through processes which are somehow time-consuming (Moguilesky and Raynaud, 1979; Parsond et al., 1980 ; Attradi and Plumbo, 1981). But the difference in the peripheral $17 \beta$-estradiol levels between the intact and cervically stimulated groups in this study did not seem likely to explain the difference in PRc concentrations between the two groups.

In conclusion, PRc concentrations in the hypothalamus strikingly fluctuate on the morning of the estrous day, which may give a basis either for the effectiveness of progesterone treatment for inducing psp or for the circadian characteristics of PRL surges. The search for the entity that controls the concentration of PRc in hypothalamic neurons in an acute fashion is now under way.

\section{Acknowledgement}

The authors are indebted to Dr. Junzo Kato for his invaluable advice concerning the measurement of PRc.

\section{References}

Attardi, B. and Palumbo, L. A. (1981). Effects of Nafoxidine on the luteinizing hormone surge: temporal distribution of estrogen receptors and induction of cytoplasmic progestin receptors in the hypothalamus-preoptic area, pituitary, and uterus of the immature rat. Endocrinology 109, 13651374.

Clemens, J. A., E. B. Smaltig and B. D. Sawyer (1976). Studies on the role of preoptic area in the control of reproductive function in the rat. Endocrinology 99, 728-735.

Everett, J. W. (1963). Pseudopregnancy in the rat from brief treatment with progesterone: effect of isolation. Nature 198, 695-696.

Freeman, M. E. and J. A. Banks (1980). Hypothalamic sites which control the surges of prolactin secretion induced by cervical stimulation. Endocrinology 106, 668-673.

Freeman, M. E., M. S. Smith, S. J. Nazian and J. D. Neill (1974). The pattern of prolactin secretion during pseudopregnancy in the rat. ibid. 90, 12921294.

Ginsburg, M., B. D. Greenstein, N. J. MacLusky, I. D. Morris and P. J. Thomas (1974). An improved method for the study of high-affinity steroid binding: oestradiol binding in brain and pituitary. Steroids 23, 773-792.

Kato, J. and T. Onouchi (1979). Nuclear progesterone receptors and characterization of cytosol receptors in the rat hypothalamus and anterior hypophysis. J. Steroid Biochem. 11, 845-854.

Lauine, V. N., R. I. Khylchevskaya and B. S. McEwen (1974). Oestrogen effects on brain and pituitary enzyme activities. J. Biochem. 23, 925-934.

Lowry, O. H., N. J. Rosenbrough, A. L. Farr and R. J. Randall (1951). Protein measurement with the Folin phenol reagent. J. Biol. Chem. 193, 265275.

MacLusky, N. T. and B. S. McEwen (1980). Progesterone receptors in rat brain: distribution and properties of cytoplasmic progestin-binding sites. Endocrnology 106, 192-201.

Moguilesky, N. J. and B. S. MacEwen (1980). Estrogen-sensitive binding sites in the female rat brain and pituitary. Brain Res. 164, 165-172.

Murakami, N., M. Takahashi and Y. Suzuki (1979). Indispensable role of peripheral progesterone level 
for the occurrence of prolactin surges in pseudopregnant rats. Biol. Reprod. 21, 263-268.

Murakami, N., M. Takahashi and Y. Suzuki (1980). Induction of pseudopregnancy and prolactin surges by a single injection of progesterone. ibid. 22, 253-258.

Murakami, N., M. Takahashi, Y. Suzuki and K. Homma (1982). Responsiveness of dispersed rat luteal cells to luteinizing hormone and prolactin during the estrous cycle and early pseudopregnancy. Endocrinology 111, 500-508.

Nishihara, M. and M. Takahashi (1983). Effects of active immunization against estradiol-17 $\beta$ on LH and testosterone in male rats. Biol. Reprod. 29, (in press)

Parsons, B., N. J. MacLusky, L. Krey, D. W. Pfaff and B. S. McEwen (1980). The temporal relationship between estrogen-inducible progestin receptors in the female rat brain and the time course of estrogen activation of mating behavior. Endocrinology 107, 774-779.

Raynaud, J. P. (1977). R5020, a tag for the progestin receptor. In: Progresterone Receptors in Normal and Neoplasmic Tissues (W. L. McGuire, J. P. Raynaud and E. E. Baulieu eds.), Raven Press, New York. pp. 9-21.

Scatchard, G. (1949). The attraction of proteins for small molecules and ions. Ann. N. Y. Acad. Sci. $51,660-672$.

Smith, M. S. and J. D. Neill (1976). Termination of the two daily surges of plasma prolactin initiated by mating in the rat. Endocrinology 98, 696-701. Takahashi, M., N. Murakami, H. Naito and Y. Suzuki (1980). Blockade of pseudopregnancy in the rat by treatment with antiprogesterone serum. Biol. Reprod. 22, 423-429. 